University of South Carolina

Scholar Commons

Faculty Publications

Electrical Engineering, Department of

$12-1996$

\title{
Detection for a Statistically-Known, Time-Varying Dispersive Channel
}

David W. Matolak

University of South Carolina - Columbia, matolak@cec.sc.edu

S. G. Wilson

Follow this and additional works at: https://scholarcommons.sc.edu/elct_facpub

Part of the Digital Communications and Networking Commons, Signal Processing Commons, and the Systems and Communications Commons

\section{Publication Info}

Postprint version. Published in IEEE Transactions on Communications, Volume 44, Issue 12, 1996, pages 1673-1683.

(C) IEEE Transactions on Communications, 1996, IEEE

Matolak, D., Wilson, S. (1996). Detection for a Statistically-Known, Time-Varying Dispersive Channel. IEEE Transactions on Communications, 44(12), 1673-1683.

http://dx.doi.org/10.1109/26.545897

This Article is brought to you by the Electrical Engineering, Department of at Scholar Commons. It has been accepted for inclusion in Faculty Publications by an authorized administrator of Scholar Commons. For more information, please contact digres@mailbox.sc.edu. 


\title{
Detection for a Statistically Known, Time-Varying Dispersive Channel
}

\author{
David W. Matolak, Member, IEEE, and Stephen G. Wilson, Member, IEEE
}

\begin{abstract}
Detection for the statistically known channel (SKC) is aimed at obtaining good performance in situations where our statistical knowledge of a time-varying channel is good, and where other equalization/detection schemes are either too complex to implement, or their performance is limited due to the rapidity of channel fading, or where we are simply unable to perform channel estimation. By using a statistical characterization of the channel, we develop a new detector that performs maximumlikelihood sequence estimation (MLSE) (given the channel model) on blocks of $N$ symbols. Both symbol-spaced and fractionally spaced samples are used, to obtain two different detectors, that are generalizations of those devised for optimal block schemes on nondispersive channels. The detector that uses fractionally spaced samples is shown to outperform the detector that uses symbol-spaced samples. The performance of both appears to approach that of the corresponding known channel (KC) detector as the blocklength increases. We also numerically evaluate the SKC detector performance under conditions where the channel parameters (statistics) are incorrectly estimated, and show that the fractionally spaced detector is fairly robust to modeling errors. Finally, we devise a sliding block algorithm, for use when transmitting more than $N$ symbols.
\end{abstract}

\section{INTRODUCTION}

W E CONSIDER communication over mildly dispersive, time-varying channels, as typified by mobile cellular systems, e.g., the North American IS-54 standard. Our inquiry concerns the description and performance of an optimal block detector of the data symbols, armed with a known statistical description of the physical propagation channel. This detector provides an alternate processor structure to those that first estimate (and track) the channel evolution, then use these estimates in a "known-channel" (KC) sequence estimator. Under the assumptions of the model, our detector performs at least as well as the "decoupled" receivers which operate on the same amount of received data [1].

We show that fractionally-sampled (FS) receivers, using two samples/data symbol, dramatically outperform single-sample processors on this time-varying channel, despite the slow variation of the channel. The FS detector achieves diversity order two, which is the maximum obtainable with the assumed two-ray physical channel. Furthermore, the fractionally spaced

Paper approved by R. Kennedy, the Editor for Data Communications Mod ulation \& Signal Design of the IEEE Communications Society. Manuscript received October 17, 1995; revised April 15, 1996. This paper was presented at the 46th IEEE Vehicular Technology Conference, Atlanta, GA, April, 1996

D. W. Matolak was with Loral Communication Systems, Salt Lake City, UT 84116-2588 USA. He is now with The MITRE Corporation, McLean, VA 22102-3431 USA.

S. G. Wilson is with the Department of Electrical Engineering, University of Virginia, Thornton Hall, Charlottesville, VA 22903 USA

Publisher Item Identifier S 0090-6778(96)09027-7. detector exhibits good robustness to modeling errors. We also demonstrate that as blocklength increases, error probability approaches that of a $\mathrm{KC}$ sequence detector, showing that the detector is implicitly "learning" the unknown channel in effect for the block (to within a phase rotation). The performance is already comparable for blocklengths of a few data symbols.

The detector structure is based on the estimator/correlator [2], but is the first application (that we know of) to dispersive channels. In [3], the authors used this same idea on a nondispersive time-varying Rayleigh-fading channel. The authors of [4] applied the same principles to maximum-likelihood (ML) detection of continuous phase modulation (CPM) signals, also on a nondispersive channel. Related work is that of [5] and [6], for the additive white Gaussian noise (AWGN) channel, wherein the authors also observed improved performance [approaching that of differentially-encoded, coherently-detected phase shift keying (PSK)] with increasing blocklength. More recently, in [7], the authors formulated and solved the detection problem for a nondispersive channel in a coded system.

Other recent related work is that of [8], in which an "innovations" approach is used. This approach derives a trellis-based detector appropriate for very rapidly fading channels--for the modest fading rates considered here the trellis complexity would likely be prohibitively large. Our sliding block scheme, which is the extension of our block detector to situations in which we transmit sequences of length greater than $N$ symbols, can be cast as a sparse tree-search detection scheme (see Section IV). It thus represents a tree-search counterpart to the trellis search of [8].

We begin by describing the channel model in Section II. In Section III, we use this channel model in developing our detector, a MLSE given our knowledge of the channel. Section IV develops some expressions for pairwise sequence error probability, and bounds for average sequence and symbol error probability. Also included in this section are computer simulation results which support the analysis, and results for a sliding block algorithm - the practical extension of the block detector. Section V contains conclusions.

\section{Continuous and Discrete-Time Channel Model}

Fig. 1 shows a block diagram of the complex envelope form of the continuous-time model. In this figure, $h_{T}(\tau)$ and $h_{R}(\tau)$ represent the (time-invariant) impulse responses of the transmitter and receiver filters, respectively, and $\tau$ is the delay variable. The data input is the $M$-ary PSK sequence $\left\{d_{n}\right\}$, which is differentially encoded to yield the sequence $\left\{a_{n}\right\}$. The additive noise $n(t)$ is white and Gaussian, and $c(\tau ; t)$ is 


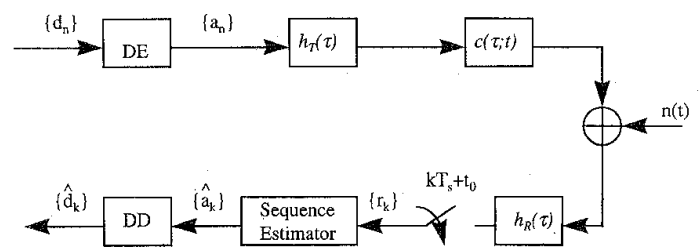

Fig. 1. Block diagram of the communication system under consideration, in complex envelope form.

the complex envelope of the time-varying impulse response of the actual radio channel. Specifically, $c(\tau ; t)$ represents the response of the channel at time $t$ to an impulse input at time $t-\tau$. The blocks labeled "DE" and "DD" are the differential encoder and decoder, respectively. The data sequence is input synchronously with a symbol rate $1 / T$, and the output $r(t)$ is sampled at times $k T_{s}+t_{0}$, where $T_{s} \leq T$ and $t_{0}$ is the sample timing offset, or sampling phase. Although we specifically address only constant-amplitude signaling schemes, namely PSK, all of our analysis is directly applicable to quadratureamplitude modulation (QAM) schemes as well.

Since all the filters are presumed linear, we can represent the composite filter, as seen by the input $\left\{a_{n}\right\}$ and the output $r(t)$, as the convolution (with respect to $\tau$ ) of $h_{T}(\tau), c(\tau ; t)$, and $h_{R}(\tau)$. We denote this resultant impulse response $f(\tau ; t)=$ $h_{T}(\tau) * c(\tau ; t) * h_{R}(\tau)$. Samples (at times $k T_{s}$ ) of the received waveform $r(t)$ are then

$$
r\left(k T_{s}\right)=r_{k}=\sum_{n} a_{n} f\left(k T_{s}-n T ; k T_{s}\right)+w_{k}
$$

where $w_{k}=w\left(k T_{s}\right)$ represents the $k$ th sample of the (assumed white) Gaussian noise process $w(t)=n(t) * h_{R}(t)$. Here, we are concerned with two values of the sampling period $T_{s}: T$ and $T / 2$. The first case is denoted $T S$, and the second $F S$, for $T$-spaced, and fractionally-spaced, respectively. Using the commutative property of convolution, we have

$$
r_{k}=\sum_{n=0}^{L} a_{l-n} f_{i}(k)+w_{k}
$$

where for the TS case, we have $l=k$, and $i=n$; and for the FS case, $l=\lceil k / 2\rceil$, and $i=2 n+m$, with $m=(k+1)$ $\bmod 2$, i.e., $m=1$ for $k$ even, and $m=0$ for $k$ odd. The duration of the equivalent discrete-time response, determined by the duration of the responses $c(\tau ; t), h_{T}(\tau)$, and $h_{R}(\tau)$, is essentially finite for practical channels, i.e., we neglect the response beyond some maximum duration, denoted $L T$. We note that, assuming the response $f(\tau ; t)$ is strictly bandlimited, the FS sampled sequence represents a set of sufficient statistics via the Nyquist theorem; this does not, in general, apply to the TS sampled sequence. Fig. 2 shows the TS equivalent channel model, and Fig. 3 shows the FS equivalent channel model for the case of $2(L+1)=6$, i.e., $L=2$.

The channel impulse response $c(\tau ; t)$ is the complex envelope of the actual RF bandpass physical channel impulse response. Since for practical cases this response is unknown and time-varying, we model it statistically. As is typical, we model $c(\tau ; t)$ as a complex zero-mean Gaussian process (in $t$ ) [9]; the envelope of $c(\tau ; t)$ then has Rayleigh statistics. This

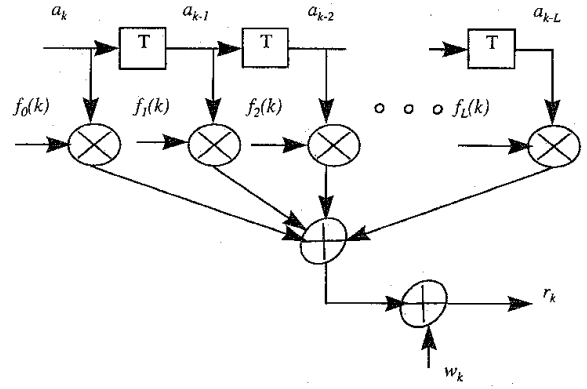

Fig. 2. Tapped delay line model of the equivalent discrete-time channel for Fig. 1, for the TS case. The blocks containing " $T$ " denote delays of $T \mathrm{~s}$.

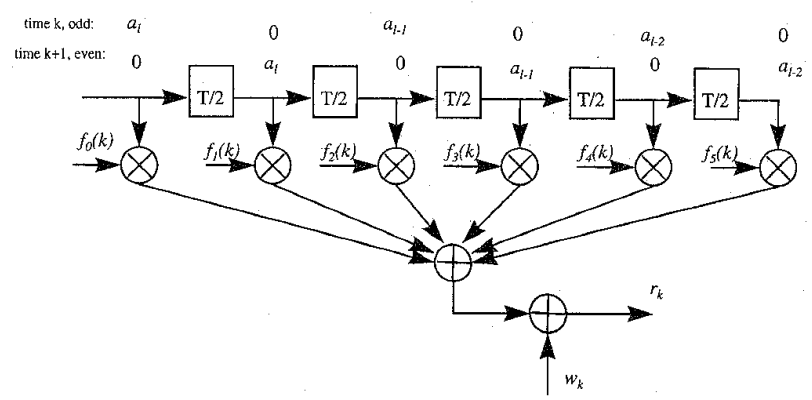

Fig. 3. Tapped delay line model of the equivalent discrete-time channel for Fig. 1, for the FS case and a channel impulse response length of $3 T$. The blocks containing " $T / 2$ " denote delays of $T / 2 \mathrm{~s}$, and $l=\lceil k / 2\rceil$, and $\lceil x\rceil$ denotes the smallest integer $\geq x$.

also implies that $f(\tau ; t)$ is complex zero-mean Gaussian in $t$. We also adopt the WSSUS model [10]. We thus characterize the length (in $\tau$ ) of $c(\tau ; t)$ by the channel delay spread $T_{M}$, and the rapidity of its time variation by the Doppler spread $f_{D}$. Specifying $T_{M}$ and $f_{D}$ then allows specification of the equivalent response duration and the time rate of change of the discrete time filter taps $f_{n}(k)$. For the two cases of concern (TS and FS), we then have the complete equivalent discrete time models. For this study, concerned with digital mobile radio (cellular), we follow the (time-division multiple-access, TDMA) North American digital standard denoted $I S-54$ [11], and model the cascade of transmitter and receiver filters as raised-cosine (in frequency), with rolloff equal to 0.35 .

The form and parameters adopted for $c(\tau ; t)$ are based on several factors: reported measurements [12]-[15], analysis and simulation [16], [17], and expedience. We focus on a near worst-case channel in terms of $T_{M}$ and $f_{D}$. For a worst case value of $T_{M}$, we use $20 \mathrm{~s}$ [12]. With the IS-54 symbol duration of $T=1 / 24000 \cong 41.7 \mu \mathrm{s}$, this corresponds to roughly $T_{M}=T / 2$, i.e., the echo delay is half a symbol duration. For the impulse response shape, we use a "double-spike" [16], spaced by $T_{M}: c(\tau ; t)=c_{0}(t) \delta(\tau)+c_{1}(t) \delta\left(\tau-T_{M}\right)$ where $E\left[\left|c_{0}(t)\right|^{2}\right]=E\left[\left|c_{1}(t)\right|^{2}\right]=0.5$ so that the average energy of the channel is normalized to one. Since the uncorrelated scattering assumption applies, the (complex Gaussian) random processes $c_{0}(t)$ and $c_{1}(t)$ are independent (we "observe" them, via $c(\tau ; t)$ at different delays).

With our chosen channel response, the response $f(\tau ; t)$ is then

$$
f(\tau ; t)=c_{0}(t) h(\tau)+c_{1}(t) h\left(\tau-T_{M}\right)
$$


where $h(\tau)$ is the full raised-cosine response, equal to the convolution of $h_{T}(\tau)$ and $h_{R}(\tau)$. To obtain the discrete-time equivalent tap weights, $f_{n}(k)$ of (2), we set $\tau$ equal to $n T_{s}$ and $t$ equal to $k T_{s}$ (assuming the sampling phase $t_{0}=0$ ). For the TS case, we have, with $T_{s}=T$,

$$
\begin{aligned}
& f_{0}^{\mathrm{TS}}(k)=c_{0}(k) h(0)+c_{1}(k) h(T / 2) \\
& f_{n}^{\mathrm{TS}}(k)=c_{1}(k) h((2 n-1) T / 2), \quad n=1,2, \cdots
\end{aligned}
$$

and where the symmetry of $h(\tau)$ was used. For the TS case, we truncate the number of taps to $L+1=3$. The normalized average tap energies are then $E\left[\left|f_{0}\right|^{2}\right]=0.7717, E\left[\left|f_{1}\right|^{2}\right]=$ 0.2136 , and $E\left[\left|f_{2}\right|^{2}\right]=0.0147$.

In the FS case, with $\tau=n T / 2$ and $t=k T / 2$ in (3), we obtain

$$
\begin{array}{rlr}
f_{0}^{\mathrm{FS}}(k) & =c_{0}(k) h(0)+c_{1}(k) h(T / 2) \\
f_{1}^{\mathrm{FS}}(k) & =c_{0}(k) h(T / 2)+c_{1}(k) h(0) & \\
f_{2 n}^{\mathrm{FS}}(k) & =c_{1}(k) h((2 n-1) T / 2) & n= \pm 1, \pm 2, \cdots \\
f_{2 n+1}^{\mathrm{FS}}(k) & =c_{0}(k) h((2 n+1) T / 2) & n= \pm 1, \pm 2, \cdots .
\end{array}
$$

As in the TS case, we retain only the most significant taps, yielding $2(L+1)=6$. The average (normalized) tap energies here are $E\left[\left|f_{-2}\right|^{2}\right]=E\left[\left|f_{3}\right|^{2}\right]=0.00735, E\left[\left|f_{-1}\right|^{2}\right]=$ $E\left[\left|f_{2}\right|^{2}\right]=0.1068$, and $E\left[\left|f_{0}\right|^{2}\right]=E\left[\left|f_{1}\right|^{2}\right]=0.38585$. In both the TS and FS cases, we chose the minimum number of taps such that the truncated response contained at least $98 \%$ of the energy of the untruncated response.

For specifying the dynamic behavior of the complex discrete-time Gaussian processes $c_{0}(k)$ and $c_{1}(k)$ (samples of $c_{0}(t)$ and $\left.c_{1}(t)\right)$ as functions of time, we again rely on measurements and analytical convenience. As modeled in [18], the autocorrelation of the continuous-time processes $c_{0}(t)$ and $c_{1}(t)$ is $r_{c}(\tau)=J_{0}\left(2 \pi \tau f_{D}\right)$, where $J_{0}(x)$ is the zeroth order Bessel function of the first kind. For our system model, for a vehicle speed of $30 \mathrm{~m} / \mathrm{s}(67.1 \mathrm{mph})$ and a carrier frequency of $900 \mathrm{MHz}$, the maximum Doppler shift $f_{D} \cong 90 \mathrm{~Hz}$. For simulation purposes, we approximate the autocorrelation by something more easily synthesized, namely, the inverse Fourier transform of a Chebyshev Type-I magnitude-squared frequency response. For time separations $\tau \leq 50 T$, the Chebyshev filter yields a very good approximation to the desired autocorrelation [1].

In [1] we derived the auto- and cross-correlation functions for the tap weights $f_{n}(k)$ for both the TS and FS cases. These correlations are functions of the physical channel tap autocorrelation $\left(r_{c}(\tau)\right.$ mentioned above) and the filter response $h(\tau)$. We use the correlation functions in the following sections in developing our detection scheme and in analyzing its performance.

\section{RECEIVER STRUCTURE AND DETECTION SCHEME}

\section{A. Statistical Description of the Received Sequence}

We now obtain a statistical description of the received sequence $\left\{r_{k}\right\}$, to which we apply MLSE principles. Thus, we are essentially performing optimal sequence detection given our knowledge of the channel. The MLSE approach for the statistically known channel yields formulas for the sequence metrics, which are used to rank the sequences. An important point here is that the derived metrics apply to sequences of a specific length, which we denote by $N$. For longer sequences, a modification of the algorithm is described in detail in [1] (for which some results are presented in Section IV-C).

In the discussion in the previous section, we noted that the equivalent channel taps are characterized as complex Gaussian random processes, with zero mean, and covariance that depends on the assumed correlations of the physical channel taps. Since our noise process $\left\{w_{k}\right\}$ is also zero-mean complex Gaussian (and white), it follows that our received sequence $\left\{r_{k}\right\}$, given a hypothesized transmitted sequence $\left\{a_{k}\right\}$, is also zero-mean complex Gaussian.

Here, we follow the development in [3], which addresses the nondispersive channel; see also [19]. It is worth stating that our dispersive case reduces to the nondispersive case when we set $f_{n}(k)=0$ for all $n \neq 0$ (or, equivalently, setting $c_{1}(k)=0$ ); thus, ours is a generalization of the nondispersive case. We organize the sequences into vectors of length $N$, and form the necessary data and covariance matrixes to obtain a complete statistical description of the received sequence. We focus here on the TS case; the analogous FS case is developed in detail in [1]. The received sequence in the TS case is expressed as follows [see (2)]

$$
\boldsymbol{r}_{N}=\left(r_{1}, r_{2}, \cdots, r_{N}\right)^{T}
$$

and the transmitted sequence is

$$
\boldsymbol{a}_{N}=\left(a_{1}, a_{2}, \cdots, a_{N}\right)^{T}
$$

where the $T$ denotes transpose, i.e., the vectors are defined as column vectors.

The probability density function (pdf) for the received sequence, given some transmitted sequence, is denoted $p\left(\boldsymbol{r}_{N} \mid \boldsymbol{a}_{N}\right)$. As noted, the pdf is complex Gaussian

$$
p\left(\boldsymbol{r}_{N} \mid \boldsymbol{a}_{N}\right)=\frac{1}{\pi \cdot\left|C\left(\boldsymbol{a}_{N}\right)\right|} \cdot \exp \left(-\boldsymbol{r}_{N}^{H} C^{-1}\left(\boldsymbol{a}_{N}\right) \boldsymbol{r}_{N}\right)
$$

where $C\left(a_{N}\right)$ denotes the covariance matrix of $r_{N}$ given $a_{N},|\cdot|$ denotes determinant, and the superscript $H$ denotes Hermitian (conjugate transpose). The elements of $C\left(\boldsymbol{a}_{\boldsymbol{N}}\right)$, abbreviated $C_{a}$, are obtained by taking the expectation $E\left[\boldsymbol{r}_{N}\right.$. $\left.\boldsymbol{r}_{N}^{H} \mid \boldsymbol{a}_{N}\right]$. To do this, we first express the received sequence $r_{N}$ as follows:

$$
\boldsymbol{r}_{N}=A \boldsymbol{f}_{N}+\boldsymbol{w}_{N}
$$

where $w_{N}$ is defined analogously to $r_{N}$ in (10), and the $N(L+1) \times 1$ vector $\boldsymbol{f}_{\boldsymbol{N}}$ is defined by

$$
f_{N}=\left[f_{0 N}\left|f_{1 N}\right| \cdots \mid f_{L N}\right]^{T}
$$

where $f_{i} \boldsymbol{N}$ is defined as the vector of time samples of the $i$ th tap weight

$$
f_{i N}=\left(f_{i}(1), f_{i}(2), \cdots, f_{i}(N)\right)^{T}
$$

for $i=0,1, \cdots, L$. The $N \times N(L+1)$ matrix $A$ is also defined as a partition

$$
A=\left[A_{0}\left|A_{1}\right| \cdots A_{L}\right]
$$


where each of the $A_{i}$ matrixes is a (square) diagonal data matrix, whose elements are defined as

$$
A_{i}=\operatorname{diag}\left(a_{-i+1}, a_{-i+2}, \cdots, a_{-i+N}\right)
$$

for $i=0,1, \cdots, L$. We note that forming the $A_{i}$ 's requires that we have knowledge of the additional symbols $a_{0}, a_{-1}, \cdots$. If we are operating in the middle of an indefinitely long sequence, the subscripts denote time in reference to the current time. Otherwise, we can simply transmit a few $(L)$ known symbols as an initial training sequence.

We define next the covariance matrix of the actual discretetime channel taps

$$
K_{f}=E\left[f_{N} \cdot f_{N}^{H}\right]
$$

The matrix $K_{f}$ is an $N(L+1) \times N(L+1)$ matrix. The elements of $K_{f}$ are the auto and cross-correlations of the equivalent channel taps, as defined in [1]. For example, for the case where $L=2, K_{f}$ is a $3 N \times 3 N$ matrix

$$
K_{f}=\left[\begin{array}{lll}
K_{00} & K_{01} & K_{02} \\
K_{10} & K_{11} & K_{12} \\
K_{20} & K_{21} & K_{22}
\end{array}\right]
$$

where the Hermitian and Toeplitz $N \times N$ submatrixes are defined as

$$
K_{n m}=E\left[\boldsymbol{f}_{n N} \cdot \boldsymbol{f}_{m N}^{H}\right]=\left[k_{i j}^{n m}\right] \quad n, m=0,1, \cdots, L
$$

with

$$
k_{i j}^{n n}=r_{n}^{\mathrm{TS}}(j-i) \quad n=0,1, \cdots, L
$$

and

$$
k_{i j}^{n m}=\rho_{n m}^{\mathrm{TS}}(j-i) \quad n, m=0,1, \cdots, L
$$

where $r_{n}^{\text {TS }}(x)=E\left[f_{n}(k) f_{n}^{*}(k-x)\right]$ is the auto-correlation of the $n$th equivalent channel tap, and $\rho_{n m}^{\text {TS }}(x)=E\left[f_{n}(k) f_{m}^{*}(k-\right.$ $x)]$ is the cross-correlation between the $n$th and $m$ th taps.

Using the definitions above, we can now express the covariance matrix $C_{\boldsymbol{a}}$ as follows:

$$
C_{\boldsymbol{a}}=A K_{f} A^{H}+\sigma_{w}^{2} I_{N}
$$

where $\sigma_{w}^{2}$ is the variance of the sequence $\left\{w_{k}\right\}$, and $I_{N}$ is the $N \times N$ identity matrix. (The nonwhite noise case can be accounted for by replacing $\sigma_{w}^{2} I_{N}$ by the covariance of the noise sequence.) Thus, the elements of $C_{a}$ depend on the data sequence $\left\{a_{k}\right\}$, the channel tap correlations, and the noise variance. Specifically, we have for $c_{i j}$, the $i j$ th element of $C_{\boldsymbol{a}}$

$$
c_{i j}=\sum_{n=0}^{L} \sum_{m=0}^{L} a_{i-n} a_{j-m}^{*} E\left[f_{n}(i) f_{m}^{*}(j)\right]+\sigma_{w}^{2} \delta_{i j}
$$

where the expectation becomes either $r_{n}^{\mathrm{TS}}(i-j)$ or $\rho_{n}^{\mathrm{TS}}(i-j)$. The function $\delta_{i j}$ is the Kronecker delta, equal to zero unless $i=j$, for which it equals one. We now have a complete description of the pdf in (12).

\section{B. The ML Sequence (Block) Estimator for the Statistically Known Channel}

As is often done when the pdf is an exponential form, we take the (negative of the) natural logarithm of (12). This is an acceptable operation since the logarithm is a monotonic function of its argument, and hence its use will not change the ranking of the sequences. This results in the following as the maximum-likelihood sequence estimator (MLSE) $\hat{a}$ :

$$
\hat{\boldsymbol{a}}=\underset{\boldsymbol{a}}{\arg \min } \Lambda(\boldsymbol{a}, \boldsymbol{r})
$$

where the sequence metrics are

$$
\Lambda(\boldsymbol{a}, \boldsymbol{r})=\ln \left|C_{\boldsymbol{a}}\right|+\boldsymbol{r}^{H} C_{\boldsymbol{a}}^{-1} \boldsymbol{r}
$$

which we will abbreviate as

$$
\Lambda(\boldsymbol{a}, \boldsymbol{r})=b(\boldsymbol{a})+q(\boldsymbol{a}, \boldsymbol{r})
$$

where $b(\boldsymbol{a})$ denotes the hypothesis-dependent bias term, and $q(\boldsymbol{a}, \boldsymbol{r})$ denotes the hypothesis-and-received-sequencedependent Hermitian quadratic form. (We have discarded the constant involving $\pi$, since it is the same for all sequences.) Equations (25) and (26) constitute the MLSE rule for the statistically known dispersive channel.

The expression in (26) requires that a set of $M^{N}$ covariance matrixes (and their inverses) and a set of $M^{N}$ biases, for the $M^{N}$ sequence hypotheses, be pre-computed and stored before transmission. We also require knowledge of the average signalto-noise ratio. Some reduction in computation can be obtained through the use of symmetry (e.g., noting that $C_{a}=C_{-a}$ ), but this computation and storage nonetheless places a limit on the maximum feasible value of $N$. This issue is discussed in the next section, where we introduce a scheme for sequences of length greater than $N$. In addition, since $C_{a}$ is equal to $C_{-a}$, the metrics for sequences $\boldsymbol{a}$ and $-\boldsymbol{a}$ are identical. We use differential encoding and decoding to resolve this ambiguity.

The actual implementation of the computation of (26) can be considerably simplified by a different representation of the covariance matrixes. This is accomplished by a Cholesky factorization [20] of the covariance matrixes, as follows:

$$
C_{\boldsymbol{a}}=\tilde{L}_{\boldsymbol{a}} \tilde{U}_{\boldsymbol{a}}
$$

Then, for the inverse of $C_{a}$, we have

$$
C_{\boldsymbol{a}}^{-1}=\tilde{U}_{\boldsymbol{a}}^{-1} \tilde{L}_{\boldsymbol{a}}^{-1}=U_{\boldsymbol{a}} U_{\boldsymbol{a}}^{H}
$$

where $\tilde{U}_{\boldsymbol{a}}$ and $U_{\boldsymbol{a}}$ are upper triangular, and $\tilde{L}_{\boldsymbol{a}}$ and $U_{\boldsymbol{a}}^{H}$ are lower triangular. Using this form for $C_{a}^{-1}$, the TS metric expression becomes

$$
\begin{aligned}
\Lambda^{\mathrm{TS}}(\boldsymbol{a}, \boldsymbol{r}) & =\ln \left[\prod_{m=1}^{N} u_{m m}^{-2}\right]+\boldsymbol{r}^{H} U_{\boldsymbol{a}} U_{\boldsymbol{a}}^{H} \boldsymbol{r} \\
& =-2 \sum_{m=1}^{N} \ln \left(u_{m m}\right)+\sum_{m=1}^{N}\left|\sum_{i=0}^{m-1} u_{m-i, m}^{*} r_{m-i}\right|^{2}
\end{aligned}
$$

where $u_{i, j}$ is the $i j$ th element of $U_{\boldsymbol{a}}$. The first sum is the bias $b(\boldsymbol{a})$, and the second sum is the quadratic form $q(\boldsymbol{a}, \boldsymbol{r})$. We note 
that when $N$ is greater than the fading process memory $\tau(\cong$ $\left.1 / f_{D}\right)$, the matrix $U_{\boldsymbol{a}}$ is banded, and the metric computation can be simplified. In fact, when $U_{\boldsymbol{a}}$ is banded a trellis can be formed, as in [3] and [8]. In our more slowly fading case, where the fading process memory $\tau$ is many (perhaps hundreds of) symbols, the trellis can become prohibitively large (roughly $M^{\tau}$ states). We concentrate on smaller values of $N$, where $U_{\boldsymbol{a}}$ is not banded, for our block-based detector.

Next, we consider in more detail the quadratic form part of the sequence metric. By factoring out the first coefficient $u_{m, m}^{*}$ and pulling $r_{m}$ out of the inner sum, we obtain

$$
\begin{aligned}
q(\boldsymbol{a}, \boldsymbol{r}) & =\sum_{m=1}^{N}\left|u_{m, m}\right|^{2}\left|r_{m}+\sum_{i=1}^{m-1}\left(\frac{u_{m-i, m}^{*}}{u_{m, m}^{*}}\right) r_{m-i}\right|^{2} \\
& =\sum_{m=1}^{N} \eta_{m}\left|r_{m}-\sum_{i=1}^{m-1} v_{m, i} r_{m-i}\right|^{2} \\
& =\sum_{m=1}^{N} \eta_{m}\left|r_{m}-\hat{r}_{m}\right|^{2} \\
& =\sum_{m=1}^{N} q_{m}(\boldsymbol{a}, \boldsymbol{r})
\end{aligned}
$$

where the constants $\eta_{m}$ and $v_{m, i}$, and the sum $\hat{r}_{m}$ are implicitly defined in (31).

The metric increment $q_{m}(\boldsymbol{a}, \boldsymbol{r})$ is also implicitly defined by (31). From this equation, we make the observation that the quadratic form portion of the metric increment $q_{m}(\boldsymbol{a}, \boldsymbol{r})$, computed at any time $m$, is a constant weighting factor $\eta_{m}$ times the magnitude-squared of a weighted sum of the past $m$ received samples. This weighted sum is the $m$ th received sample $r_{m}$, minus $\hat{r}_{m}$ of (31). The quantity $\hat{r}_{m}$ can be viewed as a linear prediction of the received symbol $r_{m}$ [3], with a prediction order $m-1$. In this case, the quadratic form part of the sequence metric becomes a weighted sum of squared prediction errors, with the coefficients of the linear predictor determined from the Cholesky factorization of the covariance matrix. These linear predictor coefficients are hypothesisdependent, and time-dependent within a block. From [20], we note that the weighting factor $\eta_{m}$ corresponds to the reciprocal of the expected value of the prediction error $e_{m-1}$ squared, i.e., the reciprocal of the prediction error variance

$$
\eta_{m}=\frac{1}{\sigma_{e}^{2}}=\frac{1}{E\left[\left|e_{m-1}\right|^{2}\right]}=\frac{1}{E\left[\left|r_{m}-\hat{r}_{m}\right|^{2}\right]} .
$$

Thus, the quadratic form metric increment $q_{m}(\boldsymbol{a}, \boldsymbol{r})$ is

$$
q_{m}(\boldsymbol{a}, \boldsymbol{r})=\frac{\left|e_{m-1}\right|^{2}}{\sigma_{e}^{2}} .
$$

Fig. 4 shows a block diagram of the receiver section (detector) that computes the sequence metrics. As shown in the figure, the detector consists of a bank of tapped delay lines (TDL's), a ROM (or RAM) for storing the precomputed weighting coefficients $\left(u_{m-i, m}^{*}\right)$ and biases, summers and squaring devices, and a decision block for selecting the estimated sequence. This figure is a parallel implementation. A serial implementation can also easily be configured. Since

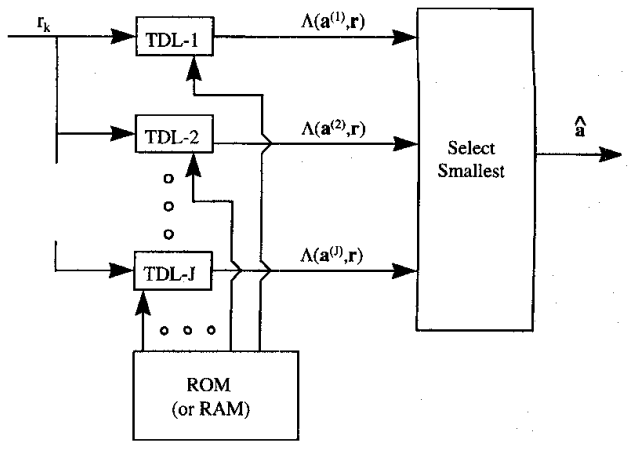

(a)

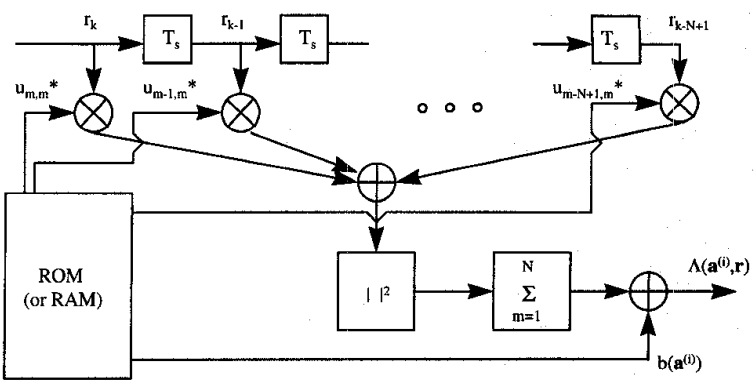

(b)

Fig. 4. Block diagram of the statistically known channel detector: (a) overall structure of the detector and (b) focus on a single tapped delay line section that computes the sequence metric for sequence $a^{(i)}$. The quantity $J=M^{N}$.

the received samples appear in the metric expressions as magnitude-squared quantities, this receiver can be viewed as a noncoherent block detector for dispersive channels.

In the nondispersive case, (obtained in our formulation by setting all $f_{n}(k)=0$ for $\left.n \neq 0\right)$, all $b(\boldsymbol{a})$ are identical and can be dropped from $\Lambda(\boldsymbol{a}, \boldsymbol{r})$. This then results in a sequence metric which is the same as that described in [7], for multiplesymbol differential detection of a block of $N$ PSK symbols received over a nondispersive fading channel. (Since in both formulations the metric is derived by maximizing $p(\boldsymbol{r} \mid \boldsymbol{a})$, this is not surprising.) As noted in [7], this metric is identical to that obtained when the channel is an AWGN channell [5], [6] (provided the fading is very slow). The receiver structure in these nondispersive cases is similar to the one derived here, but the weighting coefficients used in the tapped delay lines are simply the conjugates of the hypothesized transmitted symbols at each time $k$. As found by previous researchers [3], [5]-[7], [19], the performance of these multiple-symbol (or, block) schemes improves as the blocklength $N$ increases on the AWGN channel, approaching the performance of coherent detection as $N$ gets large. As we will see in the next section, this improved performance with increasing $N$ also holds for our dispersive channel noncoherent detector.

\section{Performance of the New Receiver}

\section{A. Pairwise Sequence Error Probability}

Using the sequence metrics, we now compute the probability that a specific incorrect sequence $a^{(i)}$ is selected, given that the 
transmitted sequence is $\boldsymbol{a}^{(t)}$. Our notation follows the TS case; the FS case is directly analogous [1]. We begin by defining this probability as $P_{2}(i, t)$

$$
P_{2}(i, t)=P\left[\Lambda\left(\boldsymbol{a}^{(i)}, \boldsymbol{r}\right)<\Lambda\left(\boldsymbol{a}^{(t)}, \boldsymbol{r}\right) \mid \boldsymbol{a}^{(t)}\right]
$$

where the metrics $\Lambda(\boldsymbol{a}, \boldsymbol{r})$, consisting of the quadratic form plus a bias, are those in (26). Suppressing the conditioning, abbreviating $C\left(\boldsymbol{a}^{(i)}\right)$ as $C_{\boldsymbol{i}}$, and $C\left(\boldsymbol{a}^{(t)}\right)$ as $C_{\boldsymbol{t}}$, and using (26) in (34), we obtain

$$
\begin{aligned}
P_{2}(i, t) & =P\left[\boldsymbol{r}^{H} C_{\boldsymbol{i}}^{-1} \boldsymbol{r}-\boldsymbol{r}^{H} C_{\boldsymbol{t}}^{-1} \boldsymbol{r}<\ln \left|C_{\boldsymbol{t}}\right|-\ln \left|C_{\boldsymbol{i}}\right|\right] \\
& =P\left[\boldsymbol{r}^{H}\left(C_{\boldsymbol{i}}^{-1}-C_{\boldsymbol{t}}^{-1}\right) \boldsymbol{r}<\ln \left(\left|C_{\boldsymbol{t}}\right| /\left|C_{\boldsymbol{i}}\right|\right)\right] \\
& =P\left[r^{H} C_{\delta}^{-1} r<\ln \left(\left|C_{\boldsymbol{t}}\right| /\left|C_{\boldsymbol{i}}\right|\right)\right] \\
& =P\left[\Delta_{q}<\Delta_{b}\right]
\end{aligned}
$$

where $\Delta_{q}$ and $\Delta_{b}$ are implicitly defined, as is the inverse difference matrix $C_{\delta}^{-1}$. The pairwise sequence error probability $P_{2}(i, t)$ is thus the probability that the random variable $\Delta_{q}$ is less than a threshold, $\Delta_{b}$. The variable $\Delta_{q}$ is a (Hermitian) quadratic form in complex Gaussian variates.

The method we use to find $P_{2}(i, t)$ is as follows. Using a well-known result for the Gaussian quadratic form, we obtain the characteristic function of $\Delta_{q}, \Phi_{\Delta}(\omega)$. We then inverse Fourier-transform this $\Phi_{\Delta}(\omega)$ to obtain the pdf of $\Delta_{q}, p_{\Delta}\left(\Delta_{q}\right)$. We finally obtain $P_{2}(i, t)$ by integrating $p_{\Delta}\left(\Delta_{q}\right)$ over the appropriate region $\left(-\infty, \Delta_{b}\right]$.

From [9, App. B], we can write the characteristic function for $\Delta_{q}, \Phi_{\Delta}(\omega)$ as

$$
\Phi_{\Delta}(\omega)=\frac{\exp \left[j \omega \mu^{H}\left(C_{\delta}-j \omega C_{t}^{*}\right)^{-1} \mu\right]}{\prod_{k=1}^{N}\left(1-j \omega \lambda_{k}\right)}
$$

where $\mu$ is the mean vector associated with $r$, and is a zero vector here, and the $\lambda_{k}$ are the eigenvalues of the matrix

$$
G=C_{\boldsymbol{t}} \cdot C_{\delta}^{-1}=C_{\boldsymbol{t}} \cdot C_{\boldsymbol{i}}^{-1}-I
$$

where $I$ is the $N \times N$ identity matrix. Since $\mu=0$, (36) becomes

$$
\Phi_{\Delta}(\omega)=\prod_{k=1}^{N} \frac{1}{1-j \omega \lambda_{k}} .
$$

We next re-express $\Phi_{\Delta}(\omega)$ in (38) as a sum using a partial fraction expansion

$$
\Phi_{\Delta}(\omega)=\sum_{k=1}^{N} \frac{\beta_{k}}{1-j \omega \lambda_{k}}
$$

with $\beta_{k}$ defined as

$$
\beta_{k}=\prod_{\substack{i=1 \\ i \neq k}}^{N} \frac{1}{1-\lambda_{i} / \lambda_{k}}
$$

We note that this expression presumes that all the eigenvalues of the matrix $G$ of (37) are distinct. This has been found to be true, empirically, for all the cases with correlated channel taps we have considered. Theoretically, repeated eigenvalues pose no problem; they yield a slightly different form for (39), with a different expression for the $\beta_{k}$ as well.

The pdf $p_{\Delta}\left(\Delta_{q}\right)$, found by a term-by-term inverse Fourier transform of (39) is then

$$
\begin{aligned}
p_{\Delta}\left(\Delta_{q}\right)= & \sum_{\substack{k=1 \\
\left\{\lambda_{k}^{+}\right\}}}^{N}\left(\frac{\beta_{k}}{\lambda_{k}}\right) \exp \left(-\Delta_{q} / \lambda_{k}\right) u\left(\Delta_{q}\right) \\
& -\sum_{\substack{k=1 \\
\left\{\lambda_{k}^{-}\right\}}}^{N}\left(\frac{\beta_{k}}{\lambda_{k}}\right) \exp \left(-\Delta_{q} / \lambda_{k}\right) u\left(-\Delta_{q}\right)
\end{aligned}
$$

where $u(\cdot)$ is the unit step function, and the sets $\left\{\lambda_{k}^{-}\right\}$and $\left\{\lambda_{k}^{+}\right\}$are

$$
\begin{aligned}
& \left\{\lambda_{k}^{-}\right\}=\left\{\lambda_{k}: \operatorname{Re}\left(\lambda_{k}\right)<0\right\} \\
& \left\{\lambda_{k}^{+}\right\}=\left\{\lambda_{k}: \operatorname{Re}\left(\lambda_{k}\right)>0\right\}
\end{aligned}
$$

where $\operatorname{Re}(x)$ is the real part of $x$. The probability $P_{2}(i, t)$ is then found by an integration of this pdf

$$
P_{2}(i, t)=\int_{-\infty}^{\Delta_{b}} p_{\Delta}\left(\Delta_{q}\right) d \Delta_{q}
$$

which results in the following expression for $P_{2}(i, t)$ :

$$
\begin{aligned}
P_{2}(i, t)= & \sum_{\substack{k=1 \\
\left\{\lambda_{k}^{-}\right\}}}^{N} \beta_{k} \exp \left(-d_{m} / \lambda_{k}^{-}\right) \\
& +\sum_{\substack{k=1 \\
\left\{\lambda_{k}^{+}\right\}}}^{N} \beta_{k}\left(1-\exp \left(-d_{p} / \lambda_{k}^{+}\right)\right) .
\end{aligned}
$$

The variables $d_{m}$ and $d_{p}$ are defined as $d_{m}=\min \left(0, \Delta_{b}\right)$, and $d_{p}=\max \left(0, \Delta_{b}\right)$, respectively.

Another expression for $P_{2}(i, t)$ is obtained if we substitute for $\Delta_{b}$ from (35). After some simplification, we have

$P_{2}(i, t)$

$$
= \begin{cases}\sum_{\substack{k=1 \\\left\{\lambda_{k}^{-}\right\}}}^{N} \beta_{k}+\sum_{\substack{k=1 \\\left\{\lambda_{k}^{+}\right\}}}^{N} \beta_{k}\left[1-\left(\frac{\left|C_{\boldsymbol{i}}\right|}{\left|C_{\boldsymbol{t}}\right|}\right)^{\lambda_{k}^{+}}\right], & \left|C_{\boldsymbol{t}}\right|>\left|C_{\boldsymbol{i}}\right| \\ \sum_{\substack{k=1 \\\left\{\lambda_{k}^{-}\right\}}}^{N} \beta_{k}\left[\left(\frac{\left|C_{\boldsymbol{i}}\right|}{\left|C_{\boldsymbol{t}}\right|}\right)^{\lambda_{k}^{-}}\right], & \left|C_{\boldsymbol{t}}\right|<\left|C_{\boldsymbol{i}}\right| \\ \sum_{\substack{\left.k=1 \\ \lambda_{k}^{-}\right\}}}^{N} \beta_{k}, & \left|C_{\boldsymbol{t}}\right|=\left|C_{\boldsymbol{i}}\right| .\end{cases}
$$

This expression shows that the sequence error probability for any two sequences can be found given the determinants of the sequences' respective covariance matrixes, and the eigenvalues of the matrix $G$, derived from these covariance matrixes and their inverses. Thus, in essence, knowledge of the covariance matrixes is sufficient to enable computation of the sequence error probability for any two sequences. Underlying 
this knowledge is of course knowledge of the channel tap statistics, and the average signal-to-noise ratio (SNR).

Unfortunately, due to the complicated way in which $P_{2}(i, t)$ is related to signal-to-noise ratio, it is impossible to determine the form of $P_{2}(i, t)$ as a function of SNR from either (45) or (46). For this, we turn to numerical evaluation.

Also of interest is the average sequence error probability $\bar{P}_{2}$, and the average symbol error probability $\bar{P}_{s}$. Using a union bound argument, we first upper bound $P_{2}(t)$, the sequence error probability given sequence $a^{(t)}$ is transmitted, as

$$
P_{2}(t) \leq \sum_{\substack{i=1 \\ i \neq t}}^{M^{N}} P_{2}(i, t)
$$

Averaging $P_{2}(t)$ over all $M^{N}$ possible transmitted sequences then yields our upper bound for average sequence error probability

$$
\begin{aligned}
\bar{P}_{2} & =\frac{1}{M^{N}} \sum_{t=1}^{M^{N}} P_{2}(t) \\
& \leq \frac{1}{M^{N}} \sum_{t=1}^{M^{N}} \sum_{\substack{i=1 \\
M^{N} \neq t}}^{M^{N}} P_{2}(i, t) .
\end{aligned}
$$

Given that sequence $\boldsymbol{a}^{(t)}$ is transmitted, the average symbol error probability $P_{s}(t)$ can be upper bounded as

$$
P_{s}(t) \leq \frac{1}{N} \sum_{\substack{i=1 \\ i \neq t}}^{M^{N}} P_{2}(i, t) w(i, t)
$$

where $w(i, t)$ is the Hamming distance between $\boldsymbol{a}^{(t)}$ and $\boldsymbol{a}^{(i)}$. The average symbol error probability $\bar{P}_{s}$ is then bounded by averaging $P_{s}(t)$ over all transmitted sequences

$$
\bar{P}_{s} \leq \frac{1}{N M^{N}} \sum_{t=1}^{M^{N}} \sum_{\substack{i=1 \\ i \neq t}}^{M^{N}} P_{2}(i, t) w(i, t) .
$$

\section{B. Analysis and Simulation Results}

We now present numerical results obtained from analysis and simulation programs for sequence and symbol error probability. In the analysis program, for sequence error probability, we compute, for all sequences, the pairwise sequence probability $P_{2}(i, t)$. This is then used in (48) to find the union upper bounds. For symbol error probability, we use $P_{2}(i, t)$ and compute the bound on $\bar{P}_{s}$ in $(50)$. These are then compared to the simulation results.

For all the results, we used the channel model described in Section II. Specifically, the channel tap correlations were generated using the two-pole Chebyshev Type-I filter mentioned in Section II, with a $3 \mathrm{~dB}$ frequency of $90 \mathrm{~Hz}$ (corresponding to a Doppler spread $f_{D} \cong 90 \mathrm{~Hz}$ ). The sampling phase $t_{0}=0$ in all cases here.

Fig. 5 shows a plot of the union upper bounds on average sequence error probability for binary antipodal signaling, blocklength $N=4$, for the TS and FS statistically known

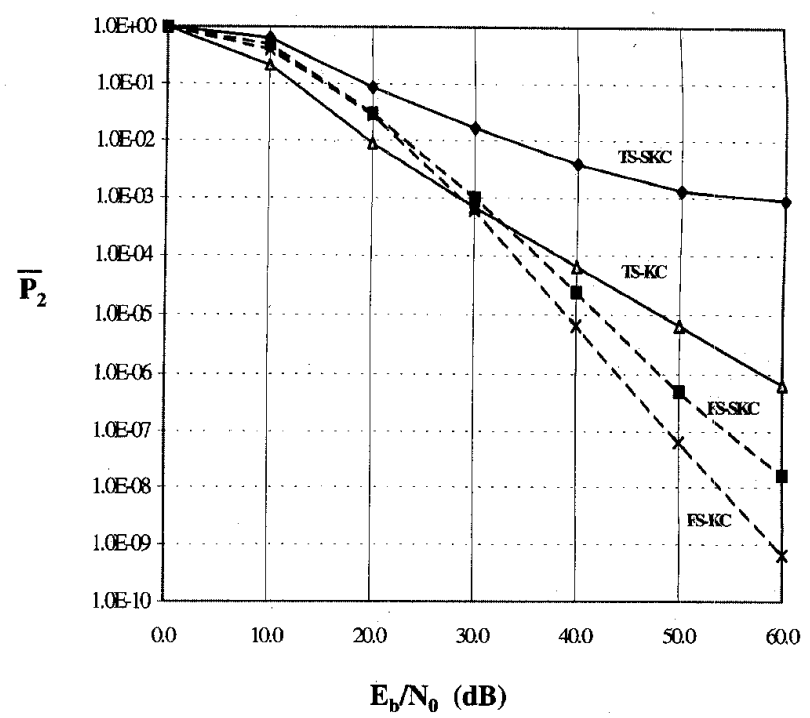

Fig. 5. Plots of union upper bounds on the average sequence error probability $\bar{P}_{2}$ versus $E_{b} / N_{0}$ for the TS and FS SKC detectors, and for the TS and FS known-channel detectors, for blocklength $N=4$, using binary PSK modulation. Channel parameters are $f_{D}=90 \mathrm{~Hz}, T_{M}=T / 2$, and $t_{0}=0$.

channel (SKC) detectors, and for the corresponding TS and FS known-channel detectors (the $\mathrm{KC}$ probabilities are derived in [1]). This is for our "baseline" case, with channel parameters $f_{D}=90 \mathrm{~Hz}, T_{M}=T / 2$, and $t_{0}=0$. As seen from this figure, the $\mathrm{KC}$ results are uniformly better than those of the SKC detectors, as one expects. Yet the FS curves are quite close, and for larger values of $N$, the SKC performance does approach that of the known channel (in both the TS and FS cases). In addition, the slopes of the SKC curves are close to the corresponding $\mathrm{KC}$ slopes, confirming the achievable diversity order in each case. An additional result regarding the $\mathrm{KC}$ detector performance was that the union bounds did not improve with increasing $N$ [1]. This same result was found in [7] for the upper bounds to sequence error probability on the nondispersive channel.

The FS detector outperforms the TS detector by a large margin at high SNR. Specifically, the FS curves approach a slope of -2 (on a $\log$ scale), which implies a diversity order of two, whereas the TS curves have at best a slope of -1 , indicating performance no better than that obtainable on a flat fading channel. Also found for this channel was that increasing the blocklength beyond four or five results in little improvement, at least in terms of the upper bound. This observation holds for the simulated results as well. Additionally, for all blocklengths an irreducible error probability (error floor) is apparent at high SNR in the TS case. The level of this error floor decreases with increasing blocklength, and is fade rate dependent. The dependence of the error floor on fade rate also holds in the nondispersive channel [21].

To corroborate the analysis, we show simulated sequence error probability results. Fig. 6 shows a plot of simulated sequence error probability for $N=4$ for the "baseline" case. The union bound results are also plotted here. As can be seen, the simulation results are in good agreement with the union 


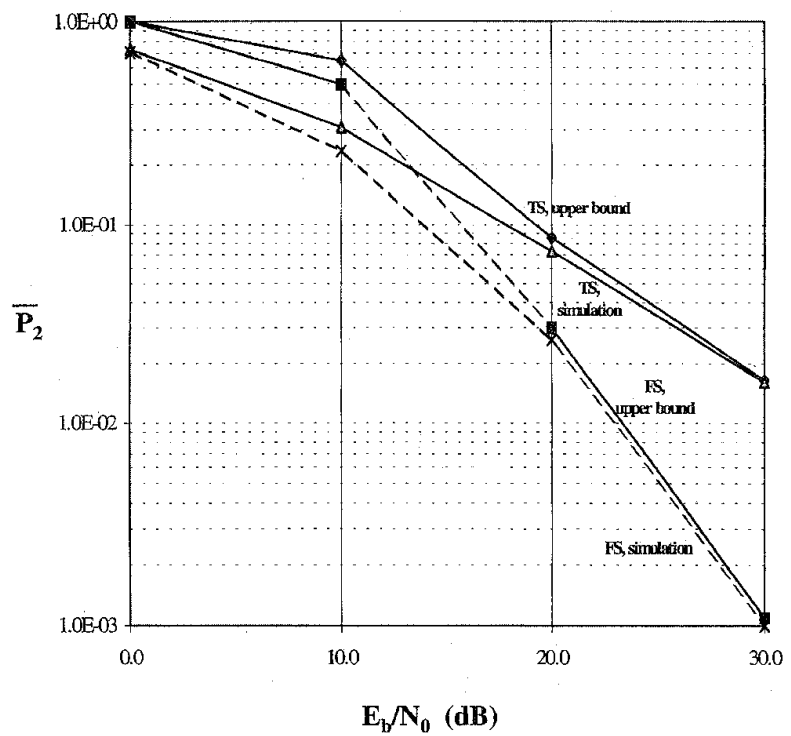

Fig. 6. Plots of the union upper bounds and simulated average sequence error probability $\bar{P}_{2}$ versus $E_{b} / N_{0}$ for the TS and FS SKC detectors, for blocklength $N=4$, and binary PSK modulation. The channel parameters are $f_{D}=90 \mathrm{~Hz}, T_{M}=T / 2$, and $t_{0}=0$.

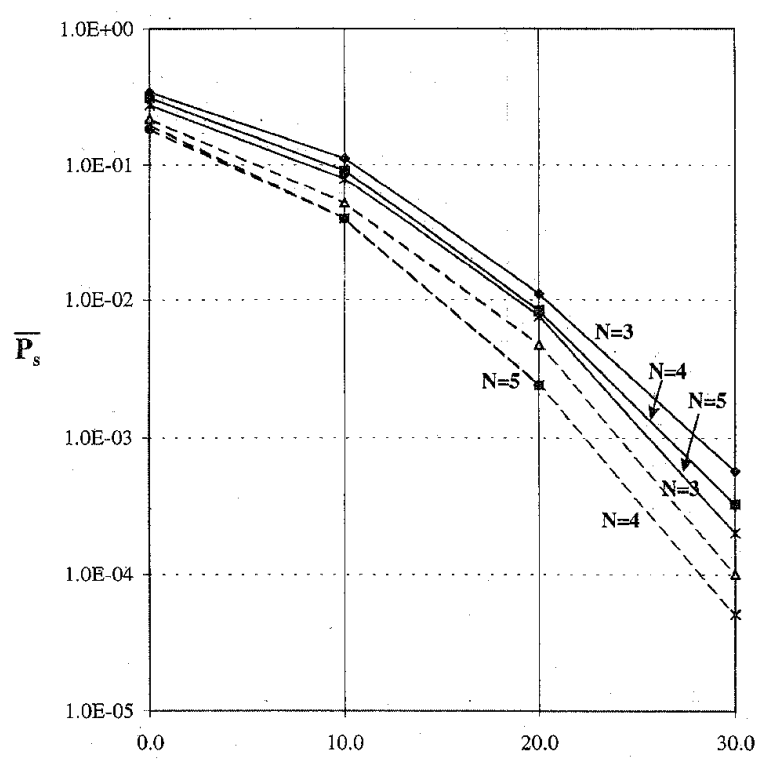

Fig. 7. Plots of simulated average symbol error probability $\bar{P}_{s}$ versus $E_{b} / N_{0}$ for the FS-SKC (solid lines) and FS-KC (dashed lines) detectors, for blocklengths $N=3,4,5$ and binary PSK modulation. The channel parameters are $f_{D}=90 \mathrm{~Hz}, T_{M}=T / 2$, and $t_{0}=0$

bound analysis, and also confirm the slopes of the two sets of curves found via analysis. This agreement also holds for other blocklengths and symbol alphabet sizes $(M)$ [1].

Fig. 7 shows a plot of simulated symbol error probability versus $E_{b} / N_{0}$. comparing the FS-SKC and FS-KC detectors. As with sequence error probability, the FS symbol error probability results are somewhat poorer than the $\mathrm{KC}$ results, but do get better with increasing blocklength. As shown in the figure, the $\mathrm{KC}$ performance does not noticeably improve beyond $N=4$, whereas the SKC performance does, lending

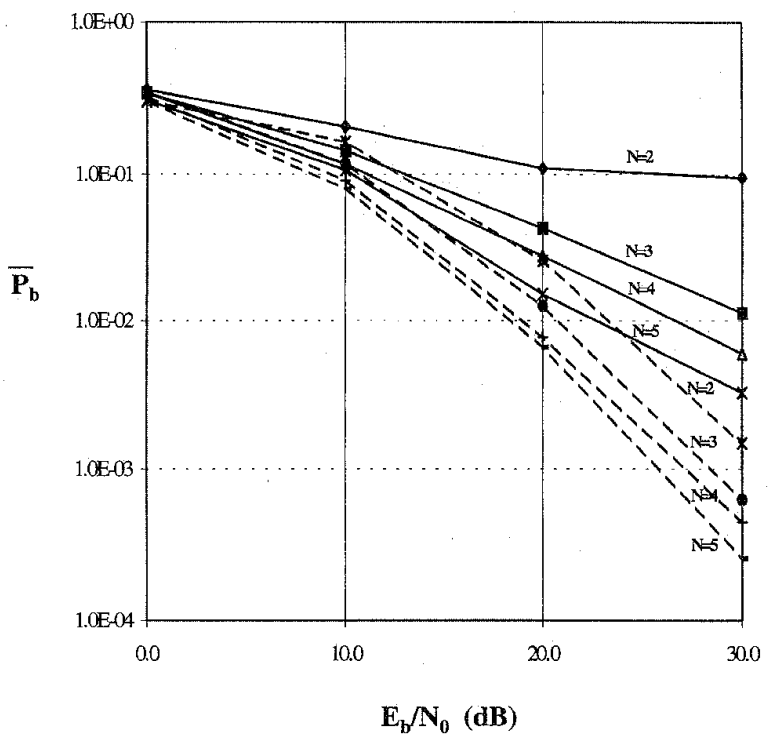

Fig. 8. Plots of simulated average BEP $\bar{P}_{b}$ (after differential decoding) versus $E_{b} / N_{0}$ for the TS (solid lines) and FS (dashed lines) SKC detectors, for blocklerigths $N=2-5$, and binary PSK modulation. The channel parameters are $f_{D}=90 \mathrm{~Hz}, T_{M}=T / 2$, and $t_{0}=0$.

credence to the claim that the SKC results approach those of the $\mathrm{KC}$ detector.

Differential encoding and decoding enables us to obtain the same final sequence output ( $\left\{d_{k}\right\}$ in Fig. 1) whether the detector selects the correct symbol sequence or its complement as the symbol sequence estimate. Thus, the bit-error probability (BEP) should be comparable to the symbol error probability. Fig. 8 shows a plot of the simulated BEP, after differential decoding, for the TS and FS cases for blocklengths from two to five for the "baseline" case. The shapes of the curves are similar to those found for symbol error probability [1], and overall, the results are quite comparable. An interesting effect is that the TS performance appears to benefit more from differential encoding and decoding than does the FS performance.

Next, we briefly discuss the sensitivity of our new receiver in the presence of an incorrect estimate of one of the system parameters. Since our receiver relies on the estimated channel parameters to compute its metrics; the sensitivity to an incorrect estimate, or "mismatch," in one or more parameters is of practical concern. Here, we consider only the effect of a change in the physical channel through a change in delay spread $T_{M}$, when this change is unknown to our receiver. Mismatches between other parameters are considered in [1].

Fig. 9 plots symbol error probability versus SNR for the TS and FS cases using a blocklength $N=4$, for three different values of actual delay spread $T_{M}: 0, T / 4$, and $T / 2$. In this plot, $\hat{f}_{D}=f_{D}=90 \mathrm{~Hz}, \hat{t}_{0}=t_{0}$, and $\hat{T}_{M}=T / 2$ for all cases, so the curves show the effect of a "mismatch" between $T_{M}$ and $\hat{T}_{M}$ only. In the TS case, the performance degrades almost catastrophically: The FS results show only a small degradation in performance when the delay spread is not correctly estimated. A point to emphasize here is that when $T_{M}$ changes, the equivalent discrete-time channel also 


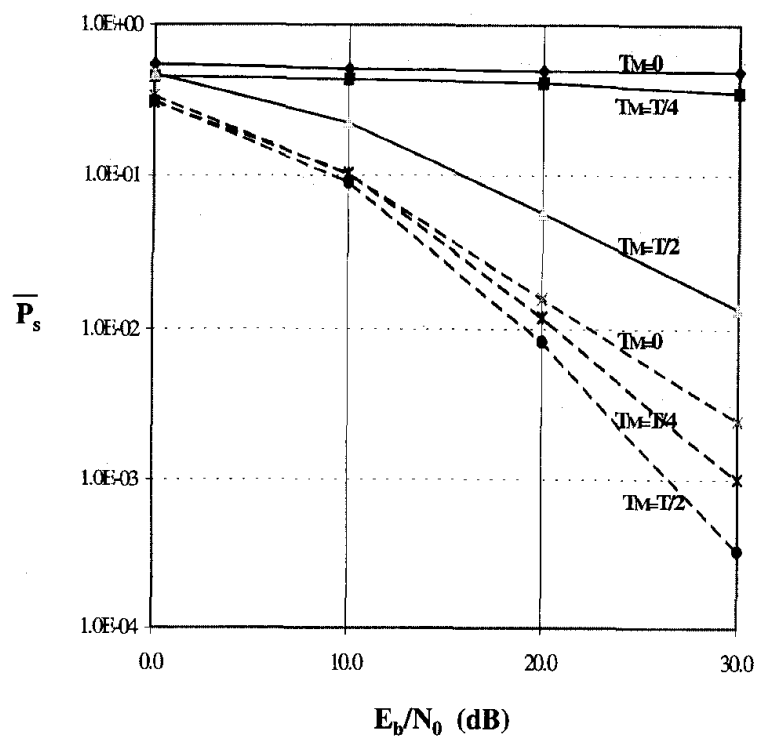

Fig. 9. Plots of simulated average symbol error probability $\bar{P}_{s}$ versus $E_{b} / N_{0}$ for the TS and FS SKC detectors, for blocklength $N=4$ and binary PSK modulation, showing the effect of mismatch between the estimated delay spread, $\hat{T}_{M}$, and the actual delay spread $T_{M}$. For all cases, $\hat{T}_{M}=T / 2$, but the actual $T_{M}$ is zero, $T / 4$, and $T / 2$. The other channel parameters are $f_{D}=90 \mathrm{~Hz}$, and $t_{0}=0$. Solid curves are TS results, dashed curves are FS results.

changes, substantially. For instance, when $T_{M}=0$, we have a nondispersive channel. For this value of delay spread, the FS performance is only slightly worse than if $\hat{T}_{M}$ were correctly estimated as zero. The effect of mismatches in Doppler spread (by more than a factor of two) and estimated signal-to-noise ratio (by $\cong 10 \mathrm{~dB}$ ) are much less significant [1], and these points illustrate the robustness of the FS-SKC detector.

\section{A Sliding Block Algorithm}

The sliding block scheme is necessary in a practical receiver because in general we will be transmitting more than a single length- $N$ block of data. We devise a scheme that uses overlapping blocks, and uses previously-decided symbols as the required feedback symbols $\left[a_{0}, a_{-1}, \cdots, a_{-L+1}\right.$ of (17)]. This will increase throughput, at the cost of some loss in performance due to decision feedback errors. Another consideration in the use of a sliding block scheme is that we now have to store $M^{N+L}$ inverse covariance factors, to account for the $L$ feedback symbols.

To aid in discussion, we introduce the following definitions: $N_{p}$ denotes the total number of symbols to be transmitted, or the number of symbols in a packet and $N_{s}$ denotes the number of symbols we slide by, or decide, in a single iteration. This is illustrated conceptually in Fig. 10, where we have shown the received and hypothesized symbol sequences for two iterations of the algorithm. The feedback symbols for the two iterations are underlined, and in this figure, $N_{s}=N-1$. We restrict $N_{s}$ to be between 1 and $N-1$. If $N_{s}=1$, we decide one symbol per iteration; if $N_{s}=N-1$, we decide $N-1$ symbols per iteration. This scheme can be cast as a sparse tree-search algorithm: for each iteration, the tree is of depth $N$ with $L$ "start-up," or feedback, symbols preceding the root

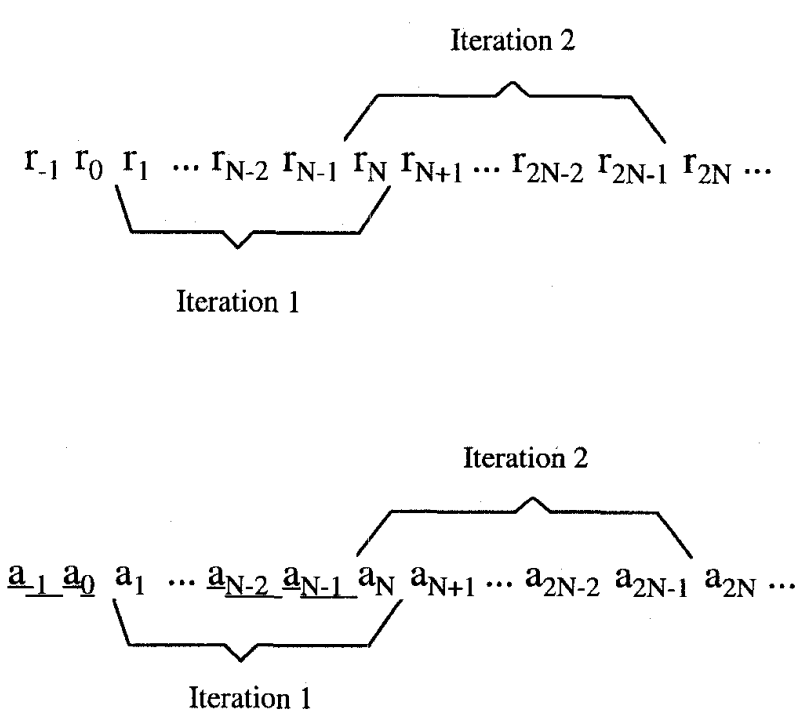

Fig. 10. Illustration of the received and hypothesized symbols used in successive iterations of the sliding block algorithm. Feedback symbols are underlined, and the parameter $N_{s}=N-1$. The start-up symbols are $a_{-1}$ and $a_{0}$.

node; after metrics are computed for each length- $N$ block, we decide $N_{s}$ symbols, and use the most recent $L$ of these "decided" symbols as feedback symbols for the next iteration. Thus, additional depth- $N$ trees are extended only from those nodes at depths $k N_{s}(k=1,2, \cdots)$ whose $L$ feedback symbols (at $k N_{s}-1, k N_{s}-2, \cdots, k N_{s}-L$ ) equal decided symbols from the previous iteration.

We next present simulation results for this sliding block algorithm. For these results, we use our "baseline" case, where $\hat{f}_{D}=f_{D}=90 \mathrm{~Hz}, \hat{T}_{0}=t_{0}=0$, and $\hat{T}_{M}=T_{M}=T / 2$. The modulation is binary PSK. For these results, we focus on BEP, after differential decoding, since the differentially decoded output sequence will be correct even if decision feedback errors have caused the detector to select the complement (generally, a phase-rotated version) of the correct symbol sequence. Fig. 11 shows results for the TS and FS cases with $N=3, N_{p}=23$, and $N_{s}=2$. We use $L=2$ initial training symbols, and so transmit $N_{p}-L=21$ information symbols per packet. Thus, throughput is $\left(N_{p}-L\right) / N_{p}$. The single block transmission results are also shown for comparison. As can be observed, the sliding block algorithm performs well, with the error probabilities in the TS case only slightly larger than those of the single-block transmission. The FS sliding-block results in this figure are actually slightly better than the single-block results. We attribute this to the relatively high $P_{b}$ of the last (third) bit in the block in the single-block transmission, which contributes much more to the average than in the sliding-block case [1]. This effect is less pronounced in the TS case.

Other practical considerations are initialization and updating of the detector parameters. As with most equalization schemes used on time-varying channels, periodic retraining is needed. For updating the inverse covariance matrixes, a recursive leastsquares algorithm, yielding time-average (instead of ensemble average) covariance matrixes could be applied. This was also proposed in [3] for the nondispersive case. 


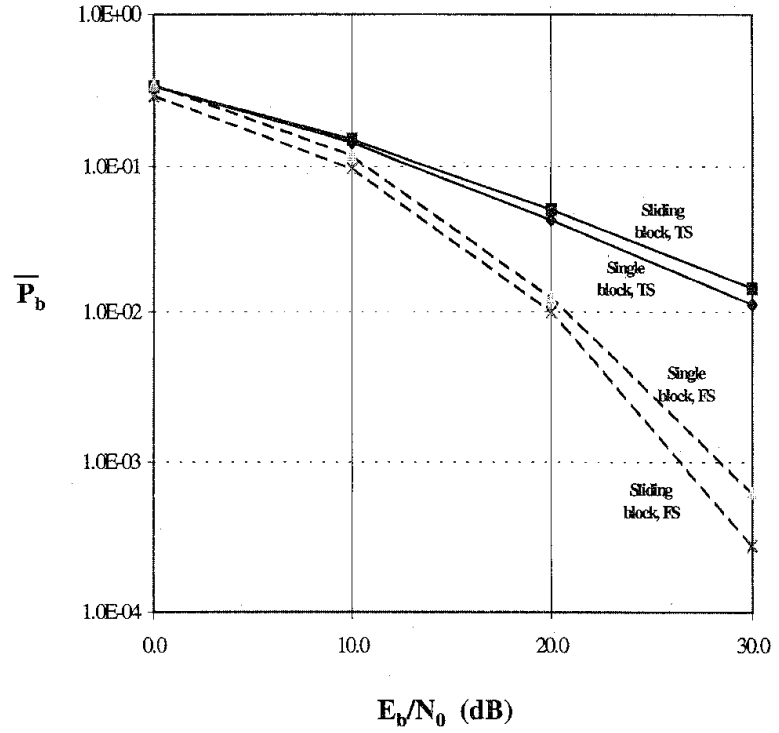

Fig. 11. Plots of simulated average bit error probability versus $E_{b} / N_{0}$ for binary PSK modulation with the TS and FS SKC detectors using the sliding-block algorithm, for blocklength $N=3$, packet length $N_{p}=23$, and decision block length $N_{s}=2$. Also shown for comparison are the corresponding single block $\left(N=3, N_{p}=N+L=5\right)$ results. The channel parameters are $f_{D}=90 \mathrm{~Hz}, T_{M}=T / 2$, and $t_{0}=0$. Solid curves are the TS results, dashed curves are the FS results.

\section{CONCLUSION}

In this paper, we developed a new detector for use on a statistically known dispersive channel. We first outlined our channel model, defined important channel parameters, and obtained a complete statistical description of the equivalent discrete-time channel and received sequence. Having this information, we then formulated the MLSE rule for sequence detection given our statistical model. This rule yielded sequence metrics consisting of a quadratic form plus a bias. Factoring the covariance matrix of the received sequence resulted in a simplification of the metrics, enabling their computation via a bank of tapped delay lines and some precomputed coefficients. The quadratic form portion of the metric was shown to be a weighted sum of linear prediction errors, resulting from a minimum mean-square error prediction of the received sequence, based on the statistical channel model. Our receiver is a generalization of the optimal block noncoherent detector for nondispersive channels.

Through operations on the quadratic form in the sequence metrics, we were able to derive an analytical expression for the pairwise sequence error probability. Using this, we described some upper bounds on average sequence and symbol error probability. We then numerically computed these bounds and compared them to simulation results. Good agreement was obtained. From these numerical results, we made several observations, the most important of which is that our new detector performs fairly well when fractionally spaced samples are used, but performs fairly poorly when symbol-spaced samples are used. The FS receiver was shown to achieve diversity at high SNR, whereas the TS receiver did not. Moreover, the FS receiver performance more quickly approached that of the known channel detector as the blocklength $N$ increased. The FS receiver was also shown to be robust in the presence of modeling errors

\section{REFERENCES}

[1] D. W. Matolak, "Variable-complexity trellis decoding, and detection for a statistically-known, time-varying, dispersive channel," Ph.D. dissertation, Univ. of Virginia, Charlottesville, VA, 1994

[2] T. Kailath, "Correlation detection of signals perturbed by a random channel,"IRE Trans. Inform. Theory, vol. 6, pp. 361-366, June 1960.

[3] G. M. Vitetta and D. P. Taylor, "Multi-sampling receivers for uncoded and coded PSK signal sequences transmitted oyer rayleigh frequencyflat fading channels," IEEE Trans. Commun., submitted for publication, Nov. 1993.

[4] J. H. Lodge and M. L. Moher, "Maximum-likelihood sequence estimation of CPM signals transmitted over Rayleigh flat-fading channels," IEEE Trans. Commun, vol. 38 , no. 6, pp. 787-794, June 1990.

[5] S. G. Wilson, J. Freebersyser, and C. Marshall, "Multi-symbol detection of MPSK," in Proc. GLOBECOM'89 Conf., Nov. 1989.

[6] D. Divsalar and M. K. Simon, "Multiple symbol differential detection of MPSK," IEEE Trans. Commun., vol. 38, no. 3, pp. 300-308, Mar. 1990

[7] — "Maximum-likelihood differential detection of uncoded and trellis coded amplitude phase modulation over AWGN and fading channels - metrics and performance," IEEE Trans. Commun., vol. 42, no. 1, pp. 76-89, Jan. 1994.

[8] X. Yu and S. Pasupathy, "Innovations-based MLSE for Rayleigh fading channels," IEEE Trans. Commun., vol. 43, no. 2/3/4, pp. 1534-1544, Feb./Mar./Apr. 1995.

[9] M. Schwartz, W. R. Bennett, and S. Stein, Communication Systems and Techniques. New York: McGraw-Hill, 1966.

[10] P. A. Bello, "Characterization of randomly time-variant linear channels," IEEE Trans. Commun. Syst., vol. CS-11, pp. 360-393, Dec. 1963.

[11] D. J. Goodman, "Second generation wireless information networks," IEEE Trans. Veh. Technol, vol. 40, no. 2, pp. 366-374, May 1991.

[12] T. S. Rappaport, S. Y. Seidel, and R. Singh, "900-MHz multipath propagation measurements for U.S. digital cellular radiotelephone," IEEE Trans. Veh. Technol, vol. 39, no. 2, pp. 132-139, May 1990

[13] S. Y. Seidel, T. S. Rappaport, S. Jain, M. L. Lord, and R. Singh, "Path loss, scattering, and multipath delay statistics in four European cities for digital cellular and microcellular radiotelephone," IEEE Trans. Veh. Technol., vol. 40, no. 4, pp. 721-730, Nov. 1991.

[14] D. C. Cox and R. P. Leck, "Correlation bandwidth and delay spread multipath propagation statistics for $910-\mathrm{MHz}$ urban mobile radio channels," IEEE Trans. Commun., vol. COM-23, no. 11, pp. 1271-1280, Nov. 1975.

[15] D. C. Cox, "Delay Doppler characteristics of multipath propagation at $910 \mathrm{MHz}$ in a suburban mobile radio environment," IEEE Trans. Antennas Propagat., vol. AP-20, no. 5, pp. 625-635, Sept. 1972.

[16] B. Glance and L. J. Greenstein, "Frequency-selective fading effects in digital mobile radio with diversity combining," IEEE Trans. Commun., vol. COM-31, no. 9, pp. 1085-1094, Sept. 1983.

[17] F. Adachi, K. Ohno, and M. Ikura, "Postdetection selection diversity reception with correlated, unequal average power Rayleigh fading signals for $\pi / 4$-shift DQPSK mobile radio," IEEE Trans. Veh. Technol., vol. 40, no. 2, pp. 199-210, May 1992.

[18] W. C. Jakes, Jr., Microwave Mobile Communications. New York: Wiley, 1974.

[19] D. Makrakis, P. Mathiopoulos, and D. P. Bouras, "Optimal decoding of coded PSK and QAM signals in correlated fast fading channels and AWGN: a combined envelope, multiple differential, and coherent detection approach," IEEE Trans. Commun., vol. 42, no. 1, pp. 63-75, Jan. 1994.

[20] S. Haykin, Adaptive Filter Theory, 2nd ed. Englewood Cliffs, NJ: Prentice-Hall; 1991

[21] J. K Cavers and P. Ho, "Analysis of the error performance of trelliscoded modulations in Rayleigh-fading channels," IEEE Trans. Commun., vol. 40, no. 1, pp. 74-83, Jan. 1992.

[22] J. G. Proakis, Digital Communications. New York: McGraw-Hill, 1989. 


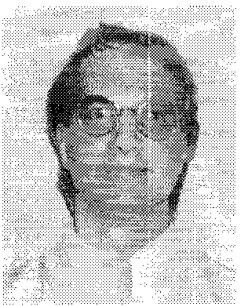

David W. Matolak (S'82-M'93) was born in Johnstown, PA, and received the B.S. degree from The Pennsylvania State University, University Park, PA in 1983, the M.S. degree from The University of Massachusetts, Amherst, MA, in 1987, and the Ph.D. degree from The University of Virginia, Charlottesville, VA, in 1995, all in electrical engineering. He was with the Rural Electrification Administration, Washington, D.C., from 1983 to 1985 , working on upgrading of specialized rural telecommunication systems. From 1985 to 1986 , he was with the UMass LAMMDA Laboratory, where he worked on the full-wave analysis, design, fabrication, and testing of planar microwave transmission lines and antennas. From 1986 to 1989 , he was with AT\&T Bell Laboratories, North Andover, MA, in the Microwave Radio Systems Development Department, where he worked on the analytical and empirical characterization of nonlinearities and their effect on QAM transmission. In 1990, he joined the University of Virginia's Communication Systems Laboratory, where his work focused on analysis of trellis coding and equalization for TDMA mobile radio systems, with additional study of CDMA. From 1994 to 1996, he was with LockheedMartin Tactical Communication Systems (formerly Loral Communications), Salt Lake City, UT, where he was Lead System Engineer on the development of a wireless local loop synchronous CDMA communication system. In 1996 he joined the MITRE Corporation, McLean, VA, where he is now working on the analysis and modeling of various digital radio communication systems. His research interests are communication over fading dispersive channels, trellis coding, CDMA, and radio channel modeling.
Stephen G. Wilson (S' $84-\mathrm{M}^{\prime} 86$ ), for a biography, see p. 1008 of the August 1996 issue of this TRANSACTIONS. 